Commun.Fac.Sci.Univ.Ank.Ser. A1 Math. Stat.

Volume 69, Number 1, Pages 952-968(2020)

DOI: $10.31801 /$ cfsuasmas.473090

ISSN 1303-5991 E-ISSN 2618-6470

http://communications.science.ankara.edu.tr

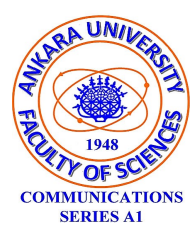

\title{
SOME GENERAL INTEGRAL INEQUALITIES FOR LIPSCHITZIAN FUNCTIONS VIA CONFORMABLE FRACTIONAL INTEGRAL
}

\author{
IMMAT İSCAN, SERCAN TURHAN, AND SELIM NUMAN
}

\begin{abstract}
In this paper, the author establishes some Hadamard-type and Bullen-type inequalities for Lipschitzian functions via Riemann Liouville fractional integral.
\end{abstract}

\section{Introduction}

Hermite-Hadamard Inequality. Let $f: I \subseteq \mathbb{R} \rightarrow \mathbb{R}$ be a convex function defined on the interval $I$ of real numbers and $a, b \in I$ with $a<b$. The following inequality

$$
f\left(\frac{a+b}{2}\right) \leq \frac{1}{b-a} \int_{a}^{b} f(x) d x \leq \frac{f(a)+f(b)}{2}
$$

holds. This double inequality is known in the literature as Hermite-Hadamard integral inequality for convex functions (see [7]). Note that some of the classical inequalities for means can be derived from (1) for appropriate particular selections of the mapping $f$.

Ostrowski's Inequality. Let $f: I \subseteq \mathbb{R} \rightarrow \mathbb{R}$ be a mapping differentiable in $I^{\circ}$, the interior of I, and let $a, b \in I^{\circ}$ with $a<b$. If $\left|f^{\prime}(x)\right| \leq M, x \in[a, b]$, then we the following inequality holds

$$
\left|f(x)-\frac{1}{b-a} \int_{a}^{b} f(t) d t\right| \leq \frac{M}{b-a}\left[\frac{(x-a)^{2}+(b-x)^{2}}{2}\right]
$$

for all $x \in[a, b]$ (see [1]).

Received by the editors: October 22, 2018; Accepted: May 02, 2020.

2010 Mathematics Subject Classification. 26A51, 26A33, 26D15.

Key words and phrases. Lipschitzian functions, Hadamard inequality, Bullen inequality, conformable fractional integral.

(C)2020 Ankara University Communications Faculty of Sciences University of Ankara-Series A1 Mathematics and Statistics 
Simpson's Inequality. Let $f:[a, b] \rightarrow \mathbb{R}$ be a four times continuously differentiable mapping on $(a, b)$ and $\left\|f^{(4)}\right\|_{\infty}=\sup _{x \in(a, b)}\left|f^{(4)}(x)\right|<\infty$. Then the following inequality holds:

$$
\left|\frac{1}{3}\left[\frac{f(a)+f(b)}{2}+2 f\left(\frac{a+b}{2}\right)\right]-\frac{1}{b-a} \int_{a}^{b} f(x) d x\right| \leq \frac{1}{2880}\left\|f^{(4)}\right\|_{\infty}(b-a)^{4}
$$

(see [3, 11] and therein).

Bullen's inequality. Suppose that $f:[a, b] \rightarrow \mathbb{R}$ is a convex function on $[a, b]$. Then we have the inequalities:

$$
\frac{1}{b-a} \int_{a}^{b} f(x) d x \leq \frac{1}{2}\left[f\left(\frac{a+b}{2}\right)+\frac{f(a)+f(b)}{2}\right]
$$

(see [5] and [16]). In what follows we recall the following definition.

Definition 1. A function $f: I \subseteq \mathbb{R} \rightarrow \mathbb{R}$ is called an $M$-Lipschitzian function on the interval $I$ of real numbers with $M \geq 0$, if

$$
|f(x)-f(y)| \leq M|x-y|
$$

for all $x, y \in I$.

For some recent results are connected with Hermite-Hadamard type integral inequalities for Lipschitzian functions, see [4, 8, 9, 17, 18. In [17, Tseng et al. established some Hadamard-type and Bullen-type inequalities for Lipschitzian functions as follows:

Theorem 2. Let $I$ be an interval in $\mathbb{R}, a \leq A \leq B \leq b$ in $I, V=(1-\alpha) a+\alpha b$, $\alpha \in[0,1]$ and let $f: I \rightarrow \mathbb{R}$ be an $L$-Lipschitzian function with $L \geq 0$. Then we have the inequality

$$
\left|\alpha f(A)+(1-\alpha) f(B)-\frac{1}{b-a} \int_{a}^{b} f(x) d x\right| \leq \frac{L V_{\alpha}(A, B)}{2(b-a)},
$$

where

$$
\begin{aligned}
& V_{\alpha}(A, B) \\
& \left\{\begin{array}{c}
(A-a)^{2}-(A-V)^{2}+(B-V)^{2}+(b-B)^{2}, \\
a \leq V \leq A \leq B \leq b, \\
(A-a)^{2}+(V-A)^{2}+(B-V)^{2}+(b-B)^{2}, \\
a \leq A \leq V \leq B \leq b, \\
(A-a)^{2}+(V-A)^{2}+(b-B)^{2}-(V-B)^{2}, \\
a \leq A \leq B \leq V \leq b
\end{array} .\right.
\end{aligned}
$$


Theorem 3. Let $I$ be an interval in $\mathbb{R}, a \leq A \leq B \leq C \leq b$ in $I, V_{1}=(1-\alpha) a+\alpha b$, $V_{2}=\gamma a+(\alpha+\beta) b, \alpha, \beta, \gamma \in[0,1], \alpha+\beta+\gamma=1$, and let $f: I \rightarrow \mathbb{R}$ be an $L$ Lipschitzian function with $L \geq 0$. Then we have the inequality

$$
\left|\alpha f(A)+\beta f(B)+\gamma f(C)-\frac{1}{b-a} \int_{a}^{b} f(x) d x\right| \leq \frac{L V_{\alpha, \beta, \gamma}(A, B, C)}{2(b-a)},
$$

where $V_{\alpha, \beta, \gamma}$ is defined as in [17, Section 3].

We give some necessary definitions and mathematical preliminaries of fractional calculus theory which are used throughout this paper.

Definition 4. Let $f \in L[a, b]$. The Riemann-Liouville fractional integrals $J_{a^{+}}^{\alpha} f$ and $J_{b^{-}}^{\alpha} f$ of order $\alpha>0$ are defined by

$$
J_{a+}^{\alpha} f(x)=\frac{1}{\Gamma(\alpha)} \int_{a}^{x}(x-t)^{\alpha-1} f(t) d t, x>a
$$

and

$$
J_{b-}^{\alpha} f(x)=\frac{1}{\Gamma(\alpha)} \int_{x}^{b}(t-x)^{\alpha-1} f(t) d t, x<b
$$

respectively, where $\Gamma(\alpha)$ is the Gamma function defined by $\Gamma(\alpha)=\int_{0}^{\infty} e^{-t} t^{\alpha-1} d t$ and $J_{a^{+}}^{0} f(x)=J_{b^{-}}^{0} f(x)=f(x)$ (see [13]).

In the case of $\alpha=1$, the fractional integral reduces to the classical integral. For some recent results connected with fractional integral inequalities, see [2, 10, 14, 15, 19]. In [15], Sarıkaya et. al. represented Hermite-Hadamard's inequalities in fractional integral forms as follows:

Theorem 5. Let $f:[a, b] \rightarrow \mathbb{R}$ be a positive function with $0 \leq a<b$ and $f \in L[a, b]$. If $f$ is a convex function on $[a, b]$, then the following inequalities for fractional integrals hold

$$
f\left(\frac{a+b}{2}\right) \leq \frac{\Gamma(\alpha+1)}{2(b-a)^{\alpha}}\left[J_{a+}^{\alpha} f(b)+J_{b-}^{\alpha} f(a)\right] \leq \frac{f(a)+f(b)}{2}
$$

with $\alpha>0$.

Definition 6. Let $\alpha \in(n, n+1], n=0,1,2, \ldots$ and set $\beta=\alpha-n$. Then the left conformable factional integral of any order $\alpha>0$ is defined by

$$
\left(I_{\alpha}^{a} f\right)(x)=\frac{1}{n !} \int_{a}^{x}(x-t)^{n}(t-a)^{\beta-1} f(t) d t,
$$


and analogously, the right conformable fractional integral of any order $\alpha>0$ is defined by

$$
\left({ }^{b} I_{\alpha} f\right)(x)=\frac{1}{n !} \int_{x}^{b}(t-x)^{n}(b-t)^{\beta-1} f(t) d t .
$$

Notice that, if $\alpha=n+1$ then $\beta=\alpha-n=1$ and hence $\left(I_{\alpha}^{a} f\right)(x)=J_{a+}^{n+1} f(x)$ and $\left({ }^{b} I_{\alpha} f\right)(x)=J_{b-}^{n+1} f(x)$. Also, if $n=0$ and $\alpha=1$ then $\beta=1$ and hence $\left(I_{\alpha}^{a} f\right)(b)=\left({ }^{b} I_{\alpha} f\right)(a)=\int_{a}^{b} f(t) d t$.

The Beta function defined as follows:

$$
B(a, b)=\frac{\Gamma(a) \Gamma(b)}{\Gamma(a+b)}=\int_{0}^{1} t^{a-1}(1-t)^{b-1} d t, a, b>0 .
$$

The Incomplete Beta function is defined by

$$
B_{x}(a, b)=\int_{0}^{x} t^{a-1}(1-t)^{b-1} d t, x \in[0,1], a, b>0,
$$

for $x=1$, the incomplete beta function coincides with the complete beta function. In [12, Set et. al. represented Hermite-Hadamard's inequalities for conformable fractional integrals as follows:

Theorem 7. Let $f:[a, b] \rightarrow \mathbb{R}$ be a function with $0 \leq a<b$ and $f \in L[a, b]$. If $f$ is a convex function on $[a, b]$, then the following inequalities for conformable fractional integrals hold:

$$
f\left(\frac{a+b}{2}\right) \leq \frac{\Gamma(\alpha+1)}{2(b-a)^{\alpha} \Gamma(\alpha-n)}\left[\left(I_{\alpha}^{a} f\right)(b)+\left({ }^{b} I_{\alpha} f\right)(a)\right] \leq \frac{f(a)+f(b)}{2} .
$$

The aim of this paper is to indicate generalizations of some integral inequalities for Lipschitzian functions via conformable fractional integral. The results are obtained in this study is a generalization of the results which are obtained in Theorem 2 and Theorem 3 by using conformable fractional integrals.

\section{A generalization of Hadamard and Ostrowski type inequalities FOR LIPSCHITZIAN FUNCTIONS VIA FRACTIONAL INTEGRALS}

Throughout this section, let $I$ be an interval in $\mathbb{R}, a \leq x \leq y \leq b$ in $I$ and let $f: I \rightarrow \mathbb{R}$ be an $M$-Lipschitzian function. In the next theorem, let $\lambda \in[0,1]$, $A=(1-\lambda) a+\lambda b$, and $A_{\alpha, \beta, n}, \alpha>0, n=0,1,2, \beta=\alpha-n$, as follows:

(1) If $a \leq A \leq x \leq y \leq b$, then

$$
A_{\alpha, \beta, n}(x, y, A)=K_{\alpha, \beta, n}(x, y, A)+L_{\alpha, \beta, n}^{*}(x, y, A) .
$$


(2) If $a \leq x \leq A \leq y \leq b$, then

$$
A_{\alpha, \beta, n}(x, y, A)=K_{\alpha, \beta, n}^{*}(x, y, A)+L_{\alpha, \beta, n}^{*}(x, y, A) .
$$

(3) If $a \leq x \leq y \leq A \leq b$, then

$$
A_{\alpha, \beta, n}(x, y, A)=K_{\alpha, \beta, n}^{*}(x, y, A)+L_{\alpha, \beta, n}(x, y, A) .
$$

where

$$
\begin{aligned}
K_{\alpha, \beta, n}(x, y, A)= & \left.(A-a)^{\alpha}[(x-a) B(\beta, n+1)-(A-a) B(\beta+1, n+1))\right], \\
K_{\alpha, \beta, n}^{*}(x, y, A)= & (A-a)^{\alpha}\left\{(x-a)\left[2 B_{\frac{x-a}{A-a}}(\beta, n+1)-B(\beta, n+1)\right]\right) \\
& \left.+(A-a)\left[B(\beta+1, n+1)-2 B_{\frac{x-a}{A-a}}(\beta+1, n+1)\right]\right\}, A \neq a, \\
K_{\alpha, \beta, n}^{*}(x, y, a)= & 0, \\
L_{\alpha, \beta, n}(x, y, A)= & (b-A)^{\alpha}[(A-y) B(n+1, \beta)+(b-A) B(n+2, \beta)], \\
L_{\alpha, \beta, n}^{*}(x, y, A)= & (b-A)^{\alpha}\left\{(y-A)\left[2 B_{\frac{y-A}{b-A}}(n+1, \beta)-B(n+1, \beta)\right]\right) \\
& \left.+(b-A)\left[B(n+2, \beta)-2 B_{\frac{y-A}{b-A}}(n+2, \beta)\right]\right\}, A \neq b, \\
L_{\alpha, \beta, n}^{*}(x, y, b)= & 0 .
\end{aligned}
$$

Theorem 8. Let $x, y, \alpha, \lambda, A, A_{\alpha, \beta, n}$ and the function $f$ be defined as above. Then we have the inequality for fractional integrals

$$
\begin{aligned}
& \left|\lambda^{\alpha} f(x)+(1-\lambda)^{\alpha} f(y)-\frac{\Gamma(\alpha+1)}{(b-a)^{\alpha} \Gamma(\alpha-n)}\left[\left(I_{\alpha}^{a} f\right)(A)+\left({ }^{b} I_{\alpha} f\right)(A)\right]\right| \\
& \leq \frac{\Gamma(\alpha+1) A_{\alpha, \beta, n}(x, y, A)}{n !(b-a)^{\alpha} \Gamma(\alpha-n)} M .
\end{aligned}
$$

Proof. Using the hypothesis of $f$, we have the following inequality

$$
\begin{aligned}
& \left|\lambda^{\alpha} f(x)+(1-\lambda)^{\alpha} f(y)-\frac{\Gamma(\alpha+1)}{(b-a)^{\alpha} \Gamma(\alpha-n)}\left[\left(I_{\alpha}^{a} f\right)(A)+\left({ }^{b} I_{\alpha} f\right)(A)\right]\right| \\
= & \frac{\Gamma(\alpha+1)}{n !(b-a)^{\alpha} \Gamma(\alpha-n)} \mid \int_{a}^{A}[f(x)-f(t)](A-t)^{n}(t-a)^{\beta-1} d t \\
& +\int_{A}^{b}[f(y)-f(t)](t-A)^{n}(b-t)^{\beta-1} d t \mid \\
\leq & \frac{\Gamma(\alpha+1)}{n !(b-a)^{\alpha} \Gamma(\alpha-n)}\left[\int_{a}^{A}|f(x)-f(t)|(A-t)^{n}(t-a)^{\beta-1} d t\right.
\end{aligned}
$$




$$
\begin{aligned}
& \left.+\int_{A}^{b}|f(y)-f(t)|(t-A)^{n}(b-t)^{\beta-1} d t\right] \\
\leq & \frac{\Gamma(\alpha+1) M}{n !(b-a)^{\alpha} \Gamma(\alpha-n)}\left[\int_{a}^{A}|x-t|(A-t)^{n}(t-a)^{\beta-1} d t\right. \\
& \left.+\int_{A}^{b}|y-t|(t-A)^{n}(b-t)^{\beta-1} d t\right] .
\end{aligned}
$$

Now using simple calculations, we obtain the following identities

$$
\int_{a}^{A}|x-t|(A-t)^{n}(t-a)^{\beta-1} d t \text { and } \int_{A}^{b}|y-t|(t-A)^{n}(b-t)^{\beta-1} d t .
$$

1. If $a \leq A \leq x \leq y \leq b$, then

$$
\begin{aligned}
\int_{a}^{A}|x-t|(A & -t)^{n}(t-a)^{\beta-1} d t \\
& \left.=(A-a)^{\alpha}[(x-a) B(\beta, n+1)-(A-a) B(\beta+1, n+1))\right] \\
& =K_{\alpha, \beta, n}(x, y, A) .
\end{aligned}
$$

and

$$
\begin{aligned}
\int_{A}^{b}|y-t|(t-A)^{n}(b-t)^{\beta-1} d t & \\
= & (b-A)^{\alpha}\left\{(y-A)\left[2 B_{\frac{y-A}{b-A}}(n+1, \beta)-B(n+1, \beta)\right]\right) \\
& \left.+(b-A)\left[B(n+2, \beta)-2 B_{\frac{y-A}{b-A}}(n+2, \beta)\right]\right\} \\
= & L_{\alpha, \beta, n}^{*}(x, y, A) .
\end{aligned}
$$

2. If $a \leq x \leq A \leq y \leq b$, then

$$
\begin{aligned}
\int_{a}^{A}|x-t|(A- & t)^{n}(t-a)^{\beta-1} d t \\
= & (A-a)^{\alpha}\left\{(x-a)\left[2 B_{\frac{x-a}{A-a}}(\beta, n+1)-B(\beta, n+1)\right]\right) \\
& \left.+(A-a)\left[B(\beta+1, n+1)-2 B_{\frac{x-a}{A-a}}(\beta+1, n+1)\right]\right\} \\
= & K_{\alpha, \beta, n}^{*}(x, y, A) .
\end{aligned}
$$


and

$$
\begin{aligned}
& \int_{A}^{b}|y-t|(t-A)^{n}(b-t)^{\beta-1} d t \\
= & (b-A)^{\alpha}\left\{(y-A)\left[2 B_{\frac{y-A}{b-A}}(n+1, \beta)-B(n+1, \beta)\right]\right) \\
& \left.+(b-A)\left[B(n+2, \beta)-2 B_{\frac{y-A}{b-A}}(n+2, \beta)\right]\right\} \\
= & L_{\alpha, \beta, n}^{*}(x, y, A) .
\end{aligned}
$$

3. If $a \leq x \leq y \leq A \leq b$, then

$$
\begin{aligned}
\int_{a}^{A}|x-t|(A-t)^{n}(t-a)^{\beta-1} d t \\
=(A-a)^{\alpha}\left\{(x-a)\left[2 B_{\frac{x-a}{A-a}}(\beta, n+1)-B(\beta, n+1)\right]\right) \\
\left.+(A-a)\left[B(\beta+1, n+1)-2 B_{\frac{x-a}{A-a}}(\beta+1, n+1)\right]\right\} \\
=K_{\alpha, \beta, n}^{*}(x, y, A),
\end{aligned}
$$

and

$$
\begin{aligned}
& \int_{A}^{b}|y-t|(t-A)^{n}(b-t)^{\beta-1} d t \\
& \quad=(b-A)^{\alpha}[(A-y) B(n+1, \beta)+(b-A) B(n+2, \beta)]=L_{\alpha, \beta, n}(x, y, A) .
\end{aligned}
$$

Using the inequality (7) and the above identities $\int_{a}^{A}|x-t|(A-t)^{n}(t-a)^{\beta-1} d t$ and $\int_{A}^{b}|y-t|(t-A)^{n}(b-t)^{\beta-1} d t$, we derive the inequality (6). This completes the proof.

Under the assumptions of Theorem 8, we have the following corollaries and remarks as follows:

Remark 9. In Theorem 8 , if we take $\alpha=\beta=1$ and $n=0$, then the inequality (6) reduces the inequality (2) in Theorem 2 under the appropriate symbols.

Corollary 10. In Theorem 8, let $\delta \in\left[\frac{1}{2}, 1\right], x=\delta a+(1-\delta) b$ and $y=(1-\delta) a+\delta b$. Then, we have the inequality

$$
\begin{aligned}
& \mid \lambda^{\alpha} f(\delta a+(1-\delta) b)+(1-\lambda)^{\alpha} f((1-\delta) a+\delta b) \\
& -\frac{\Gamma(\alpha+1)}{(b-a)^{\alpha} \Gamma(\alpha-n)}\left[\left(I_{\alpha}^{a} f\right)(A)+\left({ }^{b} I_{\alpha} f\right)(A)\right] \mid \\
\leq & \frac{\Gamma(\alpha+1) A_{\alpha, \beta, n}(\delta a+(1-\delta) b,(1-\delta) a+\delta b, A)}{n !(b-a)^{\alpha} \Gamma(\alpha-n)} M .
\end{aligned}
$$


Specially if we choose, if we take $x=y=A$, then we have Ostrowski-type inequality as follows:

$$
\begin{aligned}
& \left|\left[\lambda^{\alpha}+(1-\lambda)^{\alpha}\right] f(x)-\frac{\Gamma(\alpha+1)}{(b-a)^{\alpha} \Gamma(\alpha-n)}\left[\left(I_{\alpha}^{a} f\right)(A)+\left({ }^{b} I_{\alpha} f\right)(A)\right]\right| \\
\leq & \frac{\Gamma(\alpha+1) A_{\alpha, \beta, n}(x, y, A)}{n !(b-a)^{\alpha} \Gamma(\alpha-n)} M,
\end{aligned}
$$

where

$A_{\alpha, \beta, n}(x, y, A)=(x-a)^{\alpha+1}(B(\beta, n+1)-B(\beta+1, n+1))+(b-x)^{\alpha+1} B(n+2, \beta)$.

Remark 11. In the inequality (9), if we take $\alpha=n+1$, then the inequality (9) reduces the inequality (2.4) obtained via Riemann-Liouville fractional integrals in [10, Corollary 2.1].

Corollary 12. We have the following weighted Hadamard-type inequalities for Lipschitzian functions via conformable fractional integrals as follows:

In the inequality (8), if we take $\delta=1$, then we have

$$
\begin{aligned}
& \left|\lambda^{\alpha} f(a)+(1-\lambda)^{\alpha} f(b)-\frac{\Gamma(\alpha+1)}{(b-a)^{\alpha} \Gamma(\alpha-n)}\left[\left(I_{\alpha}^{a} f\right)(A)+\left({ }^{b} I_{\alpha} f\right)(A)\right]\right| \\
\leq & \frac{\Gamma(\alpha+1) A_{\alpha, \beta, n}(a, b, A)}{n !(b-a)^{\alpha} \Gamma(\alpha-n)} M,
\end{aligned}
$$

where

$$
\begin{aligned}
A_{\alpha, \beta, n}(a, b, A)= & (A-a)^{\alpha+1}[B(\beta+1, n+1)-B(\beta, n+1)] \\
& +(b-A)^{\alpha+1}[B(n+1, \beta)-B(n+2, \beta)],
\end{aligned}
$$

in this inequality, specially if we choose $\lambda=\frac{x-a}{b-a}$ for $x \in[a, b]$, then

$$
\begin{aligned}
& \left|\frac{(x-a)^{\alpha} f(a)+(b-x)^{\alpha} f(b)}{(b-a)^{\alpha}}-\frac{\Gamma(\alpha+1)}{(b-a)^{\alpha} \Gamma(\alpha-n)}\left[\left(I_{\alpha}^{a} f\right)(x)+\left({ }^{b} I_{\alpha} f\right)(x)\right]\right| \\
\leq & \frac{\Gamma(\alpha+1) A_{\alpha, \beta, n}(a, b, x)}{n !(b-a)^{\alpha} \Gamma(\alpha-n)} M,
\end{aligned}
$$

Corollary 13. In the inequality (9),

(i) if we choose $\lambda=\frac{1}{2}$, then

$$
\begin{aligned}
& \left|f\left(\frac{a+b}{2}\right)-\frac{2^{\alpha-1} \Gamma(\alpha+1)}{(b-a)^{\alpha} \Gamma(\alpha-n)}\left[\left(I_{\alpha}^{a} f\right)\left(\frac{a+b}{2}\right)+\left({ }^{b} I_{\alpha} f\right)\left(\frac{a+b}{2}\right)\right]\right| \\
& \leq \frac{2^{\alpha-1} \Gamma(\alpha+1) A_{\alpha, \beta, n}\left(\frac{a+b}{2}, \frac{a+b}{2}, \frac{a+b}{2}\right)}{n !(b-a)^{\alpha} \Gamma(\alpha-n)} M,
\end{aligned}
$$

where

$$
A_{\alpha, \beta, n}\left(\frac{a+b}{2}, \frac{a+b}{2}, \frac{a+b}{2}\right)
$$




$$
=\left(\frac{b-a}{2}\right)^{\alpha+1}[B(\beta, n+1)-B(\beta+1, n+1)+B(n+2, \beta)] .
$$

(ii) In the inequality (9), if we take $\lambda=\frac{1}{2}$ and $\delta=\frac{3}{4}$ then

$$
\begin{aligned}
& \mid \frac{1}{2}\left[f\left(\frac{3 a+b}{4}\right)+f\left(\frac{a+3 b}{4}\right)\right] \\
& -\frac{2^{\alpha-1} \Gamma(\alpha+1)}{(b-a)^{\alpha} \Gamma(\alpha-n)}\left[\left(I_{\alpha}^{a} f\right)\left(\frac{a+b}{2}\right)+\left({ }^{b} I_{\alpha} f\right)\left(\frac{a+b}{2}\right)\right] \mid \\
& \leq \frac{2^{\alpha-1} \Gamma(\alpha+1) A_{\alpha, \beta, n}\left(\frac{3 a+b}{4}, \frac{a+3 b}{4}, \frac{a+b}{2}\right)}{n !(b-a)^{\alpha} \Gamma(\alpha-n)} M,
\end{aligned}
$$

where

$$
\begin{aligned}
& A_{\alpha, \beta, n}\left(\frac{3 a+b}{4}, \frac{a+3 b}{4}, \frac{a+b}{2}\right) \\
& =\left(\frac{b-a}{2}\right)^{\alpha+1}\left[B_{1 / 2}(\beta, n+1)+B_{1 / 2}(n+1, \beta)-2 B_{1 / 2}(\beta+1, n+1)\right. \\
& \left.-2 B_{1 / 2}(n+2, \beta)+B(\beta+1, n+1)+B(n+2, \beta)-B(\beta, n+1)\right] .
\end{aligned}
$$

3. A generalization of Bullen and Simpson type inequalities for LIPSCHITZIAN FUNCTIONS VIA FRACTIONAL INTEGRALS

Throughout this section, let $I$ be an interval in $\mathbb{R}, a \leq x \leq y \leq z \leq b$ in $I$ and $f: I \rightarrow \mathbb{R}$ be an $M$-lipschitzian function. In the next theorem, let $\lambda+\eta+\mu=1$, $\lambda, \eta, \mu \in[0,1], A=(1-\lambda) a+\lambda b, C=\mu a+(\lambda+\eta) b$, and define $I_{\alpha, \lambda, \eta, \mu}, \alpha>0$, as follows:

(1) If $A \leq C \leq x \leq y \leq z$ or $A \leq x \leq C \leq y \leq z$, then

$$
I_{\alpha, \lambda, \eta, \mu}(x, y, z)=M_{\alpha, \lambda, \eta, \mu}(x, y, z)+N_{\alpha, \lambda, \eta, \mu}(x, y, z)+O_{\alpha, \lambda, \eta, \mu}^{*}(x, y, z) .
$$

(2) If $A \leq x \leq y \leq C \leq z$, then

$$
I_{\alpha, \lambda, \eta, \mu}(x, y, z)=M_{\alpha, \lambda, \eta, \mu}(x, y, z)+N_{\alpha, \lambda, \eta, \mu}^{*}(x, y, z)+O_{\alpha, \lambda, \eta, \mu}^{*}(x, y, z) .
$$

(3) If $A \leq x \leq y \leq z \leq C$, then

$$
I_{\alpha, \lambda, \eta, \mu}(x, y, z)=M_{\alpha, \lambda, \eta, \mu}(x, y, z)+N_{\alpha, \lambda, \eta, \mu}^{*}(x, y, z)+O_{\alpha, \lambda, \eta, \mu}(x, y, z) .
$$

(4) If $x \leq A \leq C \leq y \leq z$, then

$$
I_{\alpha, \lambda, \eta, \mu}(x, y, z)=M_{\alpha, \lambda, \eta, \mu}^{*}(x, y, z)+N_{\alpha, \lambda, \eta, \mu}(x, y, z)+O_{\alpha, \lambda, \eta, \mu}^{*}(x, y, z) .
$$

(5) If $x \leq A \leq y \leq C \leq z$, then

$$
I_{\alpha, \lambda, \eta, \mu}(x, y, z)=M_{\alpha, \lambda, \eta, \mu}^{*}(x, y, z)+N_{\alpha, \lambda, \eta, \mu}^{*}(x, y, z)+O_{\alpha, \lambda, \eta, \mu}^{*}(x, y, z) .
$$

(6) If $x \leq A \leq y \leq z \leq C$, then

$$
I_{\alpha, \lambda, \eta, \mu}(x, y, z)=M_{\alpha, \lambda, \eta, \mu}^{*}(x, y, z)+N_{\alpha, \lambda, \eta, \mu}^{*}(x, y, z)+O_{\alpha, \lambda, \eta, \mu}(x, y, z) .
$$


(7) If $x \leq y \leq A \leq C \leq z$, then

$I_{\alpha, \lambda, \eta, \mu}(x, y, z)=M_{\alpha, \lambda, \eta, \mu}^{*}(x, y, z)-N_{\alpha, \lambda, \eta, \mu}(x, y, z)+O_{\alpha, \lambda, \eta, \mu}^{*}(x, y, z)$.

(8) If $x \leq y \leq A \leq z \leq C$ or $x \leq y \leq z \leq A \leq C$, then

$I_{\alpha, \lambda, \eta, \mu}(x, y, z)=M_{\alpha, \lambda, \eta, \mu}^{*}(x, y, z)-N_{\alpha, \lambda, \eta, \mu}(x, y, z)+O_{\alpha, \lambda, \eta, \mu}(x, y, z)$.

Where

$$
\begin{aligned}
& M_{\alpha, \lambda, \eta, \mu}(x, y, z)=(A-a)^{\alpha}[(x-a) B(\beta, n+1)-(A-a) B(\beta+1, n+1)], \\
& N_{\alpha, \lambda, \eta, \mu}(x, y, z)=(C-A)^{\alpha}[(y-A) B(n+1, \beta)-(C-A) B(n+2, \beta)], \\
& O_{\alpha, \lambda, \eta, \mu}(x, y, z)=(b-C)^{\alpha}[(C-z) B(n+1, \beta)+(b-C) B(n+2, \beta)], \\
& M_{\alpha, \lambda, \eta, \mu}^{*}(x, y, z)=(A-a)^{\alpha}\left\{(x-a)\left[2 B_{\frac{x-a}{A-a}}(\beta, n+1)-B(\beta, n+1)\right]\right) \\
&\left.+(A-a)\left[B(\beta+1, n+1)-2 B_{\frac{x-a}{A-a}}(\beta+1, n+1)\right]\right\}, A \neq a(\text { or } \lambda \neq 0), \\
& M_{\alpha, 0, \eta, \mu}^{*}(x, y, z)=0 \\
& N_{\alpha, \lambda, \eta, \mu}^{*}(x, y, z)=(C-A)^{\alpha}\left\{(y-A)\left[2 B_{\frac{y-A}{C-A}}(n+1, \beta)-B(n+1, \beta)\right]\right) \\
&\left.+(C-A)\left[B(n+2, \beta)-2 B_{\frac{y-A}{C-A}}(n+2, \beta)\right]\right\}, A \neq C(\text { or } \eta \neq 0), \\
& N_{\alpha, \lambda, 0, \mu}^{*}(x, y, z)=0, \\
& O_{\alpha, \lambda, \eta, \mu}^{*}(x, y, z)=(b-C)^{\alpha}\left\{(z-C)\left[2 B_{\frac{z-C}{b-C}}(n+1, \beta)-B(n+1, \beta)\right]\right) \\
&\left.+(b-C)\left[B(n+2, \beta)-2 B_{\frac{z-C}{b-C}}(n+2, \beta)\right]\right\}, C \neq b(\text { or } \mu \neq 0), \\
& O_{\alpha, \lambda, \eta, 0}^{*}(x, y, z)=0 .
\end{aligned}
$$

Theorem 14. Let $x, y, z, \lambda, \eta, \mu, A_{1}, A_{2}, A_{\alpha, \lambda, \eta, \mu}$ and the function $f$ be defined as above. Then we have the inequality

$$
\begin{aligned}
& \mid \lambda^{\alpha} f(x)+\eta^{\alpha} f(y)+\mu^{\alpha} f(z) \\
& -\frac{\Gamma(\alpha+1)}{(b-a)^{\alpha} \Gamma(\alpha-n)}\left[\left(I_{\alpha}^{a} f\right)(A)+\left({ }^{C} I_{\alpha} f\right)(A)+\left({ }^{b} I_{\alpha} f\right)(C)\right] \mid \\
& \leq \frac{\Gamma(\alpha+1) I_{\alpha, \lambda, \eta, \mu}(x, y, z)}{n !(b-a)^{\alpha} \Gamma(\alpha-n)} M .
\end{aligned}
$$

Proof. Using the hypothesis of $f$, we have the inequality

$$
\begin{aligned}
& \mid \lambda^{\alpha} f(x)+\eta^{\alpha} f(y)+\mu^{\alpha} f(z) \\
& -\frac{\Gamma(\alpha+1)}{(b-a)^{\alpha} \Gamma(\alpha-n)}\left[\left(I_{\alpha}^{a} f\right)(A)+\left({ }^{C} I_{\alpha} f\right)(A)+\left({ }^{b} I_{\alpha} f\right)(C)\right] \mid \\
& =\frac{\Gamma(\alpha+1)}{n !(b-a)^{\alpha} \Gamma(\alpha-n)} \mid \int_{a}^{A}[f(x)-f(t)](A-t)^{n}(t-a)^{\beta-1} d t
\end{aligned}
$$




$$
\begin{aligned}
& +\int_{A}^{C}[f(y)-f(t)](t-A)^{n}(C-t)^{\beta-1} d t+\int_{C}^{b}[f(z)-f(t)](t-C)^{n}(b-t)^{\beta-1} d t \mid \\
& \leq \frac{\Gamma(\alpha+1)}{n !(b-a)^{\alpha} \Gamma(\alpha-n)}\left|\int_{a}^{A}\right| f(x)-f(t) \mid(A-t)^{n}(t-a)^{\beta-1} d t \\
& +\int_{A}^{C}|f(y)-f(t)|(t-A)^{n}(C-t)^{\beta-1} d t+\int_{C}^{b}|f(z)-f(t)|(t-C)^{n}(b-t)^{\beta-1} d t \mid \\
& \leq \frac{\Gamma(\alpha+1) M}{n !(b-a)^{\alpha} \Gamma(\alpha-n)}\left|\int_{a}^{A}\right| x-t \mid(A-t)^{n}(t-a)^{\beta-1} d t \\
& \quad+\int_{A}^{C}|y-t|(t-A)^{n}(C-t)^{\beta-1} d t+\int_{C}^{b}|z-t|(t-C)^{n}(b-t)^{\beta-1} d t \mid .
\end{aligned}
$$

Now, using simple calculations, we obtain the following identities $\int_{a}^{A}|x-t|(A-t)^{n}(t-a)^{\beta-1} d t, \int_{A}^{C}|y-t|(t-A)^{n}(C-t)^{\beta-1} d t$ and $\int_{C}^{b}|z-t|(t-C)^{n}(b-t)^{\beta-1} d t$.

(1) If $A \leq C \leq x \leq y \leq z$ or $A \leq x \leq C \leq y \leq z$, then we have

$$
\begin{aligned}
& \int_{a}^{A}|x-t|(A-t)^{n}(t-a)^{\beta-1} d t \\
& =(A-a)^{\alpha}[(x-a) B(\beta, n+1)-(A-a) B(\beta+1, n+1)] \\
& =M_{\alpha, \lambda, \eta, \mu}(x, y, z), \\
& \int_{A}^{C}|y-t|(t-A)^{n}(C-t)^{\beta-1} d t \\
& =(C-A)^{\alpha}[(y-A) B(n+1, \beta)-(C-A) B(n+2, \beta)] \\
& =N_{\alpha, \lambda, \eta, \mu}(x, y, z),
\end{aligned}
$$

and

$$
\begin{aligned}
& \int_{C}^{b}|z-t|(t-C)^{n}(b-t)^{\beta-1} d t \\
& =(b-C)^{\alpha}\left\{(z-C)\left[2 B_{\frac{z-C}{b-C}}(n+1, \beta)-B(n+1, \beta)\right]\right) \\
& \left.+(b-C)\left[B(n+2, \beta)-2 B_{\frac{z-C}{b-C}}(n+2, \beta)\right]\right\}
\end{aligned}
$$




$$
=O_{\alpha, \lambda, \eta, \mu}^{*}(x, y, z) .
$$

(2) If $A \leq x \leq y \leq C \leq z$, then we have

$$
\begin{aligned}
& \int_{a}^{A}|x-t|(A-t)^{n}(t-a)^{\beta-1} d t=M_{\alpha, \lambda, \eta, \mu}(x, y, z), \\
& \int_{A}^{C}|y-t|(t-A)^{n}(C-t)^{\beta-1} d t \\
& =(C-A)^{\alpha}\left\{(y-A)\left[2 B_{\frac{y-A}{C-A}}(n+1, \beta)-B(n+1, \beta)\right]\right) \\
& \left.+(C-A)\left[B(n+2, \beta)-2 B_{\frac{y-A}{C-A}}(n+2, \beta)\right]\right\} \\
& =N_{\alpha, \lambda, \eta, \mu}^{*}(x, y, z),
\end{aligned}
$$

and

$$
\int_{C}^{b}|z-t|(t-C)^{n}(b-t)^{\beta-1} d t=O_{\alpha, \lambda, \eta, \mu}^{*}(x, y, z) .
$$

(3) If $A \leq x \leq y \leq z \leq C$, then we have

$$
\begin{aligned}
& \int_{a}^{A}|x-t|(A-t)^{n}(t-a)^{\beta-1} d t=M_{\alpha, \lambda, \eta, \mu}(x, y, z), \\
& \int_{A}^{C}|y-t|(t-A)^{n}(C-t)^{\beta-1} d t=N_{\alpha, \lambda, \eta, \mu}^{*}(x, y, z),
\end{aligned}
$$

and

$$
\begin{aligned}
& \int_{C}^{b}|z-t|(t-C)^{n}(b-t)^{\beta-1} d t \\
& =(b-C)^{\alpha}[(C-z) B(n+1, \beta)+(b-C) B(n+2, \beta)] \\
& =O_{\alpha, \lambda, \eta, \mu}(x, y, z) .
\end{aligned}
$$

(4) If $x \leq A \leq C \leq y \leq z$, then we have

$$
\begin{aligned}
& \int_{a}^{A}|x-t|(A-t)^{n}(t-a)^{\beta-1} d t=M_{\alpha, \lambda, \eta, \mu}^{*}(x, y, z), \\
& \int_{A}^{C}|y-t|(t-A)^{n}(C-t)^{\beta-1} d t=N_{\alpha, \lambda, \eta, \mu}(x, y, z),
\end{aligned}
$$


and

$$
\int_{C}^{b}|z-t|(t-C)^{n}(b-t)^{\beta-1} d t=O_{\alpha, \lambda, \eta, \mu}^{*}(x, y, z) .
$$

(5) If $x \leq A \leq y \leq C \leq z$, then we have

$$
\begin{aligned}
& \int_{a}^{A}|x-t|(A-t)^{n}(t-a)^{\beta-1} d t=M_{\alpha, \lambda, \eta, \mu}^{*}(x, y, z), \\
& \int_{A}^{C}|y-t|(t-A)^{n}(C-t)^{\beta-1} d t=N_{\alpha, \lambda, \eta, \mu}^{*}(x, y, z),
\end{aligned}
$$

and

$$
\int_{C}^{b}|z-t|(t-C)^{n}(b-t)^{\beta-1} d t=O_{\alpha, \lambda, \eta, \mu}^{*}(x, y, z) .
$$

(6) If $x \leq A \leq y \leq z \leq C$, then we have

$$
\begin{aligned}
& \int_{a}^{A}|x-t|(A-t)^{n}(t-a)^{\beta-1} d t=M_{\alpha, \lambda, \eta, \mu}^{*}(x, y, z), \\
& \int_{A}^{C}|y-t|(t-A)^{n}(C-t)^{\beta-1} d t=N_{\alpha, \lambda, \eta, \mu}^{*}(x, y, z),
\end{aligned}
$$

and

$$
\int_{C}^{b}|z-t|(t-C)^{n}(b-t)^{\beta-1} d t=O_{\alpha, \lambda, \eta, \mu}(x, y, z) .
$$

(7) If $x \leq y \leq A \leq C \leq z$, then we have

$$
\begin{aligned}
& \int_{a}^{A}|x-t|(A-t)^{n}(t-a)^{\beta-1} d t=M_{\alpha, \lambda, \eta, \mu}^{*}(x, y, z), \\
& \int_{A}^{C}|y-t|(t-A)^{n}(C-t)^{\beta-1} d t=-N_{\alpha, \lambda, \eta, \mu}(x, y, z)
\end{aligned}
$$

and

$$
\int_{C}^{b}|z-t|(t-C)^{n}(b-t)^{\beta-1} d t=O_{\alpha, \lambda, \eta, \mu}^{*}(x, y, z) .
$$


(8) If $x \leq y \leq A \leq z \leq C$ or $x \leq y \leq z \leq A \leq C$, then we have

$$
\begin{aligned}
& \int_{a}^{A}|x-t|(A-t)^{n}(t-a)^{\beta-1} d t=M_{\alpha, \lambda, \eta, \mu}^{*}(x, y, z), \\
& \int_{A}^{C}|y-t|(t-A)^{n}(C-t)^{\beta-1} d t=-N_{\alpha, \lambda, \eta, \mu}(x, y, z),
\end{aligned}
$$

and

$$
\int_{C}^{b}|z-t|(t-C)^{n}(b-t)^{\beta-1} d t=O_{\alpha, \lambda, \eta, \mu}(x, y, z) .
$$

Using the inequality 11$)$ and the above identities $\int_{a}^{A}|x-t|(A-t)^{n}(t-a)^{\beta-1} d t$, $\int_{A}^{C}|y-t|(t-A)^{n}(C-t)^{\beta-1} d t$ and $\int_{C}^{b}|z-t|(t-C)^{n}(b-t)^{\beta-1} d t$, we derive the inequality (10). This completes the proof.

Under the assumptions of Theorem [14, we have the following corollaries and remarks as follows:

Remark 15. In Theorem 14, if we take $\alpha=\beta=1$ and $n=0$, then then the inequality (10) reduces the inequality (3) in Theorem 3 under the appropriate symbols.

Corollary 16. In Theorem 14. let $\delta \in\left[\frac{1}{2}, 1\right], x=\delta a+(1-\delta) b, y=\frac{a+b}{2}$ and $z=(1-\delta) a+\delta b$. Then, we have the inequality

$$
\begin{aligned}
& \quad \mid \lambda^{\alpha} f(\delta a+(1-\delta) b)+\eta^{\alpha} f\left(\frac{a+b}{2}\right)+\mu^{\alpha} f((1-\delta) a+\delta b) \\
& -\frac{\Gamma(\alpha+1)}{(b-a)^{\alpha} \Gamma(\alpha-n)}\left[\left(I_{\alpha}^{a} f\right)(A)+\left({ }^{C} I_{\alpha} f\right)(A)+\left({ }^{b} I_{\alpha} f\right)(C)\right] \mid \\
& \leq \frac{\Gamma(\alpha+1) I_{\alpha, \lambda, \eta, \mu}\left(\delta a+(1-\delta) b, \frac{a+b}{2},(1-\delta) a+\delta b\right)}{n !(b-a)^{\alpha} \Gamma(\alpha-n)} M .
\end{aligned}
$$

Corollary 17. In Corollary 16, if we take $\delta=1, \lambda=\mu=\frac{\theta}{2}$ and $\eta=1-\theta$ with $\theta \in[0,1]$, then we have the following weighted Bullen-type inequality for $M$ Lipschitzian functions via fractional integrals

$$
\begin{aligned}
& \mid\left(\frac{\theta}{2}\right)^{\alpha}(f(a)+f(b))+(1-\theta)^{\alpha} f\left(\frac{a+b}{2}\right) \\
& -\frac{\Gamma(\alpha+1)}{(b-a)^{\alpha} \Gamma(\alpha-n)}\left[\left(I_{\alpha}^{a} f\right)(A)+\left({ }^{C} I_{\alpha} f\right)(A)+\left({ }^{b} I_{\alpha} f\right)(C)\right] \mid \\
\leq & \frac{\Gamma(\alpha+1) I_{\alpha, \frac{\theta}{2}, 1-\theta, \frac{\theta}{2}}\left(a, \frac{a+b}{2}, b\right)}{n !(b-a)^{\alpha} \Gamma(\alpha-n)} M,
\end{aligned}
$$


where

$$
\begin{aligned}
I_{\alpha, \frac{\theta}{2}, 1-\theta, \frac{\theta}{2}}\left(a, \frac{a+b}{2}, b\right) \\
=(b-a)^{\alpha+1}\left\{\begin{array}{c}
\left(\frac{\theta}{2}\right)^{\alpha+1}[B(\beta+1, n+1)+B(n+1, \beta)-B(n+2, \beta)] \\
\left.+(1-\theta)^{\alpha+1}\left[\begin{array}{c}
B(n+2, \beta)-\frac{1}{2} B(n+1, \beta)+B_{1 / 2}(n+1, \beta) \\
-2 B_{1 / 2}(n+2, \beta)
\end{array}\right]\right\} .
\end{array}\right.
\end{aligned}
$$

Specially, in the inequality (12), if we take $n=0$ and $\alpha=\beta=1$, then the inequality (12) reduces to the following general inequality for M-Lipschitzian functions

$$
\begin{aligned}
& \left|\left(\frac{\theta}{2}\right)(f(a)+f(b))+(1-\theta) f\left(\frac{a+b}{2}\right)-\frac{1}{b-a} \int_{a}^{b} f(t) d t\right| \\
\leq & \frac{M}{4}(b-a)\left[2 \theta^{2}+(1-\theta)^{2}\right] .
\end{aligned}
$$

Remark 18. In the inequality (12), if we take $\alpha=n+1$, then the inequality (12) reduces the inequality obtained via Riemann-Liouville fractional integrals in [10, Corollary 3.2].

Remark 19. In the inequality (13), if we take $\theta=\frac{1}{3}$, then the inequality (13) reduces to the following Simpson-type inequality for $M$-Lipschitzian functions

$$
\left|\frac{1}{6}\left[f(a)+4 f\left(\frac{a+b}{2}\right)+f(b)\right]-\frac{1}{b-a} \int_{a}^{b} f(t) d t\right| \leq \frac{M}{6}(b-a) .
$$

Remark 20. In the inequality (13), if we take $\theta=\frac{1}{2}$, then the inequality (13) reduces to the following Bullen type inequality for M-Lipschitzian functions

$$
\left|\frac{1}{2}\left[\frac{f(a)+f(b)}{2}+f\left(\frac{a+b}{2}\right)\right]-\frac{1}{b-a} \int_{a}^{b} f(t) d t\right| \leq \frac{3 M}{16}(b-a) .
$$

Remark 21. In the inequality (13), if we take $\theta=0$, then the inequality (13) reduces to the following Midpoint type inequality for $M$-Lipschitzian functions

$$
\left|f\left(\frac{a+b}{2}\right)-\frac{1}{b-a} \int_{a}^{b} f(t) d t\right| \leq \frac{M}{4}(b-a) .
$$

Remark 22. In the inequality (13), if we take $\theta=1$, then the inequality (13) reduces to the following Trapezoid type inequality for $M$-Lipschitzian functions

$$
\left|\frac{f(a)+f(b)}{2}-\frac{1}{b-a} \int_{a}^{b} f(t) d t\right| \leq \frac{M}{2}(b-a) .
$$




\section{REFERENCES}

[1] Ostrowski, A. Uber die Absolutabweichung einer dierentienbaren Funktionen von ihren Integralmittelwert. Comment. Math. Helv., 10 (1938), 226-227.

[2] Dahmani, Z., On Minkowski and Hermite-Hadamard integral inequalities via fractional via fractional integration. Ann. Funct. Anal., 1 (1) (2010), 51-58.

[3] Dragomir, S.S., Agarwal R.P., Cerone, P., On Simpson's inequality and applications, J. of Inequal. Appl., 5 (2000), 533-579.

[4] Dragomir, S.S., Cho, Y.J., Kim, S.S., Inequalities of Hadamard's Type for Lipschitzian Mappings and Their Applications, J. Math. Anal. Appl., 245 (2000), 489-501.

[5] Dragomir, S.S., Pearce, C.E.M. , Selected Topics on Hermite-Hadamard Inequalities and Applications, RGMIA Monographs, Victoria University, 2000.

[6] Dragomir, S.S., Rassias, Th. M., Ostrowski type inequalities and applications in numerical integration, Kluwer Academic Publishers, Dorcdrecht, Boston, London, 2002.

[7] Hadamard, J.S., Etude sur les propiètés des fonctions entieres et en particulier d'une fontion considerer per Riemann, J. Math. Pure and Appl., 58 (1893), 171-215.

[8] Hwang, S.-R., Hsu, K.-C. and Tseng, K.-L., Hadamard-type inequalities for Lipschitzian functions in one and two variables with applications, J. Math. Anal. Appl., 405 (2013), 546554.

[9] İşcan, İ., New general integral inequalities for Lipschitzian functions via Hadamard fractional integrals. Int. J. Anal., 2014 (2014), Article ID 353924, 8 pages.

[10] İşcan,İ. , Hadamard-type and Bullen-type inequalities for Lipschitzian functions via fractional integrals, Mathematical Sciences and Applications E-Notes, 4 (1) (2016), 77-87.

[11] Sarıkaya, M.Z.,Aktan N., On the generalization of some integral inequalities and their applications, Mathematical and Computer Modelling, 54 (2011), 2175-2182.

[12] Set, E. , Akdemir, A.O. , Mumcu, I., The Hermite-Hadamard's inequality and its extentions for conformable fractioanal integrals of any order $\alpha>0$. Available online at: https://www.researchgate.net/publication/303382221.

[13] Samko, S.G., Kilbas, A.A., Marichev, O.I., Fractional Integrals and Derivatives Theory and Application, Gordan and Breach Science, New York, 1993.

[14] Sarıkaya, M.Z., Ogunmez, H., On new inequalities via Riemann-Liouville fractional integration. Abstr. Appl. Anal. 2012 (2012), Article ID 428983, 10 pages.

[15] Sarıkaya, M.Z., Set, E., Yaldız, H., Başak, N., Hermite-Hadamard's inequalities for fractional integrals and related fractional inequalities. Math. Comput. Modelling, 57 (2013), 2403-2407.

[16] Tseng, K.-L., Hwang, S.-R., Dragomir, S.S., Fejér-type inequalities (1). J. Inequal. Appl., 2010 (2010), Article ID 531976, 7 pages.

[17] Tseng, K.-L., Hwang, S.-R., Hsu, K.-C., Hadamard-type and Bullen-type inequalities for Lipschitzian functions and their applications, Comput. Math. Appl., 64 (4) (2012), 651-660.

[18] Yang, G.-S., Tseng, K.-L., Inequalities of Hadamard's Type for Lipschitzian Mappings, J. Math. Anal. Appl., 260 (1) (2001), 230-238.

[19] Zhu, C., Fečkan, M., Wang, J., Fractional integral inequalities for differentiable convex mappings and applications to special means and a midpoint formula, J. Appl. Math. Stat. Inform. 8 (2) (2012), 21-28. 
Current address: Department of Mathematics, Faculty of Arts and Sciences, Giresun University, 28200, Giresun, Turkey.

E-mail address: imdat.iscan@giresun.edu.tr; imdati@yahoo.com

ORCID Address: https://orcid.org/0000-0001-6749-0591

Current address: Department of Mathematics, Faculty of Arts and Sciences, Giresun University, 28200, Giresun, Turkey.

E-mail address: sercan.turhan@giresun.edu.tr

ORCID Address: http://orcid.org/0000-0002-4392-2182

Current address: Department of Mathematics, Faculty of Arts and Sciences, Giresun University, 28200, Giresun, Turkey.

E-mail address: selim.numan@giresun.edu.tr

ORCID Address: https://orcid.org/0000-0002-5483-6861 